\title{
Critical parameters in cultivation of experimental biofilms using the example of Pseudomonas fluorescens
}

\author{
Kirsten Reddersen $\mathbb{1}^{1} \cdot$ André Güllmar $^{2} \cdot$ Silke Tonndorf-Martini ${ }^{2} \cdot$ Bernd W. Sigusch $^{2} \cdot$ Andrea Ewald $^{3}$. \\ Thomas J. Dauben ${ }^{4} \cdot$ Karin Martin $^{5} \cdot$ Cornelia Wiegand $^{1}$
}

Received: 12 April 2021 / Accepted: 30 July 2021 / Published online: 18 August 2021

(c) The Author(s) 2021

\begin{abstract}
Formation and treatment of biofilms present a great challenge for health care and industry. About $80 \%$ of human infections are associated with biofilms including biomaterial centered infections, like infections of prosthetic heart valves, central venous catheters, or urinary catheters. Additionally, biofilms can cause food and drinking water contamination. Biofilm research focusses on application of experimental biofilm models to study initial adherence processes, to optimize physicochemical properties of medical materials for reducing interactions between materials and bacteria, and to investigate biofilm treatment under controlled conditions. Exploring new antimicrobial strategies plays a key role in a variety of scientific disciplines, like medical material research, anti-infectious research, plant engineering, or wastewater treatment. Although a variety of biofilm models exist, there is a lack of standardization for experimental protocols, and designing experimental setups remains a challenge. In this study, a number of experimental parameters critical for material research have been tested that influence formation and stability of an experimental biofilm using the non-pathogenic model strain of Pseudomonas fluorescens. These parameters include experimental time frame, nutrient supply, inoculum concentration, static and dynamic cultivation conditions, material properties, and sample treatment during staining for visualization of the biofilm. It was shown, that all tested parameters critically influence the experimental biofilm formation process. The results obtained in this study shall support material researchers in designing experimental biofilm setups.
\end{abstract}

\section{Graphical Abstract}

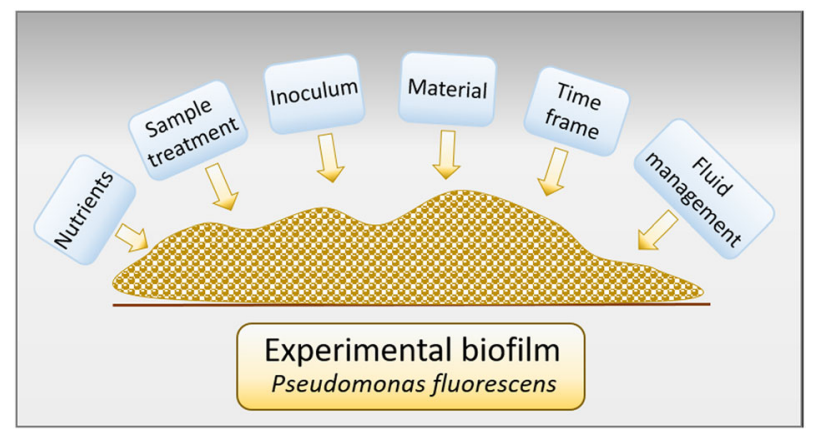

Kirsten Reddersen

kirsten.reddersen@med.uni-jena.de

1 Klinik für Hautkrankheiten, Universitätsklinikum Jena, Jena, Germany

2 Poliklinik für Konservierende Zahnheilkunde und Parodontologie, Universitätsklinikum Jena, Jena, Germany
3 Lehrstuhl für Funktionswerkstoffe der Medizin und Zahnheilkunde, Universitätsklinikum Würzburg, Würzburg, Germany

4 Lehrstuhl für Materialwissenschaft, Otto-Schott-Institut für Materialforschung, Jena, Germany

5 Hans-Knöll-Institut, Leibnitz-Institut für Naturstoff-Forschung und Infektionsbiologie, Jena, Germany 


\section{Introduction}

A biofilm is the most common form of naturally occurring microbes [1]. An estimated proportion of $99 \%$ of bacteria in natural habitats are sessile and attached as biofilms to a surface [2]. In biofilms, microorganisms are surrounded by an extracellular polymeric matrix (EPM), which accounts for about $85 \%$ of the biofilm volume [3]. The EPM creates a protective environment for the microorganisms that enhances the tolerance to harmful external conditions like antibiotics or desiccation by a factor of 10-100 compared to planktonic microorganisms [2]. This sheltering cover is considered the reason that antibiotic treatment of biofilm infections fails.

Biofilm formation plays an important role in health care and industrial settings. On the one hand, beneficial biofilms are used in the industrial production of chemicals like ethanol and vinegar. On the other hand, there are harmful effects of biofilms in production processes that cause food and drinking water contamination, metal surface corrosion, biofouling, and clogging [4]. In human health care, over $80 \%$ of all microbial infections in the body are biofilm associated [5]. This includes indwelling medical device infections of e.g. prosthetic heart valves, central venous catheters, or urinary catheters, and diseases like periodontitis, dental caries, cystic fibrosis pneumoniae, chronic urinary tract infections, infections on contact lenses, and chronic wound infections [2, 3, 6, 7]. Biomaterial implants are considered as 'microbial time bombs', since biomaterial centered infections can happen anytime and treatment is very difficult [8]. A promising approach to prevent biofilm formation on biomaterials is optimizing the physicochemical properties of biomaterial surfaces. Intelligent material design can reduce attractive forces between biomaterial and bacteria and hence, minimize initial microbial growth.

Research on biofilm development, treatment, or eradication plays a key role in antimicrobial strategies involving a variety of scientific disciplines, like medical material research, anti-infectious research, plant engineering, or wastewater treatment. Biofilm models are important tools to increase the knowledge of the underlying mechanisms of biofilm formation and to study the complex systems under controlled conditions [9]. Although a variety of in vitro and in vivo models exist [10], there is a lack of standardization of experimental protocols [11]. There is no ideal laboratory biofilm model that is representative for all biofilms and suitable for all research questions [2]. Further, the complexity of the subject makes it difficult to decide upon the appropriate biofilm model for the particular task.

In this study, a number of experimental conditions critical for material research were investigated that influence biofilm formation and biofilm stability. This includes experimental time frame, nutrient supply, inoculum concentration, static and dynamic fluid management conditions, material properties, and sample treatment for visualization. The aim of this study is to point out important factors for cultivation of experimental biofilms using the nonpathogenic model strain of Pseudomonas fluorescens and to identify critical parameters for designing experimental setups in material research.

\section{Materials and methods}

In this study, different critical parameters that influence the adherence and maturation of experimental biofilm were examined. The parameters were selected with regard to their relevance for testing bacteria/material interactions. The following critical parameters were investigated:

- Time frame of bacterial adherence

- Nutrient supply

- Inoculum concentration

- Fluid management during incubation

- Material properties

- Sample treatment for visualization

\subsection{Time frame of bacterial adherence}

Shaking overnight cultures of $P$. fluorescens (DSM 50090) were grown in Luria-Bertani-medium (LBM, Carl Roth $\mathrm{GmbH}$, Karlsruhe, Germany) at $37^{\circ} \mathrm{C}$ to mid-log phase $\left(\mathrm{OD}_{600} \approx 0.5\right)$. The culture was adjusted to an $\mathrm{OD}_{600}$ of 0.1 in diluted LBM (1:100 in $0.9 \% \mathrm{NaCl}$, Fresenius Kabi AG, Bad Homburg, Germany). Sterilized borosilicate coverslips (15 mm diameter, Karl Hecht KG, Sondheim, Germany) were placed in 12-well plates (Greiner Bio One, Frickenhausen, Germany) and incubated with $2 \mathrm{~mL}$ inoculum. Samples were incubated for $15,30,60,90$, and $120 \mathrm{~min}$ at $25^{\circ} \mathrm{C}$ in aerobic conditions. After incubation time, samples were rinsed twice with $2 \mathrm{~mL}$ phosphate buffered saline (PBS, PeloBiotech, Planegg, Germany) to remove non-adhering microorganisms, transferred to a fresh 12-well plate, and stained for $15 \mathrm{~min}$ with $1.5 \mathrm{~mL}$ SYTO9 (0.00334 mM, Invitrogen, Carlsbad, US)/ propidium iodide (PI, $0.0107 \mathrm{mg} / \mathrm{mL}$, Invitrogen, Carlsbad, US) at room temperature (RT) in the dark. After rinsing with $1 \mathrm{~mL}$ PBS, samples were incubated for $30 \mathrm{~min}$ at RT in the dark with $1.5 \mathrm{~mL}$ calcofluor white $(0.092 \mathrm{mg} / \mathrm{mL}, \mathrm{CFW}$, Sigma-Aldrich, Darmstadt, Germany). After rinsing with $1 \mathrm{~mL}$ PBS, samples were covered with mounting oil (Invitrogen, Carlsbad, US) and examined using the fluorescence microscope Axio Scope. A1 (Carl Zeiss AG, Jena, Germany) with appropriate filter blocs. Images were taken with the AxioCam MRc camera (Carl Zeiss AG, Jena, Germany) and analysed using AxioVision 4.9 software (Carl Zeiss AG, Jena, Germany). 
Adherence studies up to $72 \mathrm{~h}$ were performed in undiluted LBM with inoculum concentration of $2 \times 10^{6} \mathrm{CFU} / \mathrm{mL}$. Samples were taken after 2, 4, 6, 8, 10, 12, 16, 24, 48, and $72 \mathrm{~h}$. Medium was changed daily. Quantification with Watersoluble-tetrazolium-1 (WST-1, Roche Diagnostics, Mannheim, Germany) was carried out according to the manufacturer's instructions. Shortly, the samples were placed into fresh wells and incubated with WST-1 (10\% in PBS, SigmaAldrich, Darmstadt, Germany) for $30 \mathrm{~min}$. The absorbance was measured at $450 \mathrm{~nm}$ using a Tecan spark $20 \mathrm{M}$ plate reader (Tecan Group Ltd., Männedorf, Switzerland).

\subsection{Nutrient supply}

To study the influence of nutrient supply on biofilm adherence, LBM was applied undiluted, 1:10, 1:100, and 1:1000 diluted in $\mathrm{NaCl}(0.9 \%)$. P. fluorescens was incubated with an inoculum density of $7.6 \times 10^{5} \mathrm{CFU} / \mathrm{mL}$ in $4 \mathrm{~cm}$ diameter petri dishes with glass bottom in static conditions for $2,4,6$, 24, 48, and $72 \mathrm{~h}$. Medium was changed daily. For visualization, samples were rinsed carefully twice with $2 \mathrm{~mL} \mathrm{NaCl}$ (0.9\%), stained with PI ( $8 \mu \mathrm{M}$; Carl Roth $\mathrm{GmbH}$, Karlsruhe, Germany) in paraformaldehyde (PFA, 4\%, Carl Roth $\mathrm{GmbH}$, Karlsruhe, Germany) over night at $4{ }^{\circ} \mathrm{C}$ in the dark. After incubation, samples were washed twice with $2 \mathrm{~mL}$ $\mathrm{NaCl}(0.9 \%)$ and overlaid with $\mathrm{NaCl}(0.9 \%)$. Samples were examined with a confocal laser scanning microscope LSM 510 Meta (Carl Zeiss AG, Jena, Germany) equipped with an EC Plan-Neofluar 20x/0.50 M27 objective (Carl Zeiss AG, Jena, Germany). Propidium iodide labeled bacteria were excited with a DPSS laser $(561 \mathrm{~nm})$, and their emission passed a 574-nm longpass filter.

\subsection{Inoculum concentration}

P. fluorescens was incubated on $15 \mathrm{~mm}$ diameter borosilicate glass coverslips (Karl Hecht KG, Sondheim, Germany) under static conditions. Inoculum concentration was $2 \times 10^{3}, 2 \times 10^{4}, 2 \times 10^{5}$, and $2 \times 10^{6} \mathrm{CFU} / \mathrm{mL}$ in LBM. Samples were taken after 1, 2, 4, 24, 48, and $72 \mathrm{~h}$. Bacteria were quantified by determination of colony forming units on Columbia-agar plates $+5 \%$ sheep blood (bioMérieux, Nürtingen, Germany) after separation and serial dilution.

\subsection{Fluid management}

To compare static and dynamic growth conditions, shaking overnight cultures of $P$. fluorescens in LBM were adjusted to OD 0.5 and diluted 1:100 in LBM. $1 \mathrm{~mL}$ inoculum was incubated in 24 well microtiter plates (MTP, Greiner Bio One, Frickenhausen, Germany) in static and dynamic conditions using a wobble shaker (Titramax 100; Heidolph Instruments $\mathrm{GmbH} \& \mathrm{CO}$. KG, Schwabach, Germany) at $200 \mathrm{rpm}$.
Samples were taken at 2, 4, 6, 24, 48, and $72 \mathrm{~h}$. Medium was changed after 24 and $48 \mathrm{~h}$. Bacteria were quantified by determination of colony forming units after separation and serial dilution.

The influence of sample rinsing on biofilm growth was examined by incubating $15 \mathrm{~mm}$ diameter borosilicate coverslips (Karl Hecht KG, Sondheim, Germany) with inoculum concentrations of $2 \times 10^{7} \mathrm{CFU} / \mathrm{mL}$ for 24,48 , and $72 \mathrm{~h}$. The biofilm was rinsed once with $2 \mathrm{~mL}$ LBM before quantification and during medium change after 24 and $48 \mathrm{~h}$. Quantification was done with WST-1 (10\%) as described before.

\subsection{Material properties}

Borofloat glass coverslips (Borofloat ${ }^{\circledR}$ B33, Jena 4H Engineering $\mathrm{GmbH}$, Jena, Germany), Nunc ${ }^{\mathrm{TM}}$ Thermanox $^{\mathrm{TM}}$ polyester (Fisher Scientific GmbH, Schwerte, Germany), and poly-L-lysin coated coverslips (Corning ${ }^{\circledR}$ BioCoat $^{\mathrm{TM}}$ Poly-L-Lysine, Corning Life Sciences B.V., Amsterdam, The Netherlands) were incubated in 24 well MTP (Greiner bio One, Frickenhausen, Germany) with $1 \mathrm{~mL}$ inoculum of $1 \times 10^{6} \mathrm{CFU} / \mathrm{mL}$ for 24,48 , and $72 \mathrm{~h}$. Samples were stained with $1.5 \mathrm{~mL}$ crystal violet solution (1\%, Sigma-Aldrich, Darmstadt, Germany) for $10 \mathrm{~min}$. After three washing steps with $1 \mathrm{~mL}$ sterile distilled water the remaining dye was solved by SDS (1\%, Sigma-Aldrich, Darmstadt, Germany) and absorbance was measured at $590 \mathrm{~nm}$ using a Spekol 1200 reader (Analytik Jena, Jena, Germany).

\subsection{Sample treatment for visualization}

Drying effects during sample treatment were visualized with $24 \mathrm{~h}$ biofilm on borosilicate glass coverslips (Karl Hecht KG, Sondheim, Germany) in 12-well MTPs. Samples were incubated with an inoculum of $\mathrm{OD}_{600} 0.1$ in $\mathrm{LBM}$ diluted 1:100 in $\mathrm{NaCl}(0.9 \%)$. Staining was done with SYTO9/PI/CFW as described in chapter 2.1. To examine the influence of possible drying effects for visualization of the biofilm during sample treatment, on the one hand samples were kept consequently with a liquid film during staining procedure by treating each well separately, on the other hand removal of liquid during staining was done for the whole plate, enabling individual samples to fall dry for a short time.

\subsection{Statistics}

Experiments were performed in duplicate and each sample was measured in three replicates.

All values presented are expressed as means \pm SD. Oneway analysis of variance was carried out to determine statistical significances (Microsoft ${ }^{\circledR}$ Excel 2016). Significant 


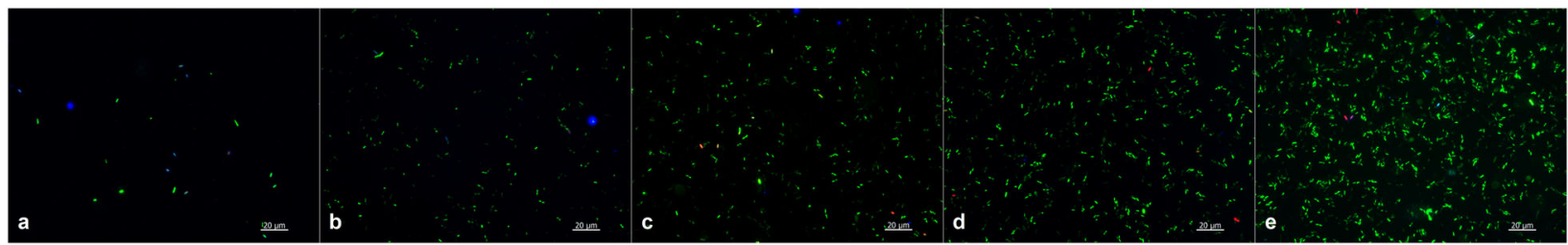

Fig. 1 Adherence of $P$. fluorescens to the borosilicate glass substrate was determined after a $15 \mathrm{~min}$, b $30 \mathrm{~min}$, c $60 \mathrm{~min}$, d $90 \mathrm{~min}$, and e 120 min incubation in LBM 1:100 diluted in $0.9 \% \mathrm{NaCl}$. Samples were rinsed after incubation and between staining steps to select only adhering microorganism. Staining of the biofilm was done with SYTO9/PI/CFW
Fig. 2 Biomass of $P$. fluorescens biofilm after up to $72 \mathrm{~h}$ incubation was quantified by cell proliferation reagent WST-1. Data are given as mean \pm standard deviation

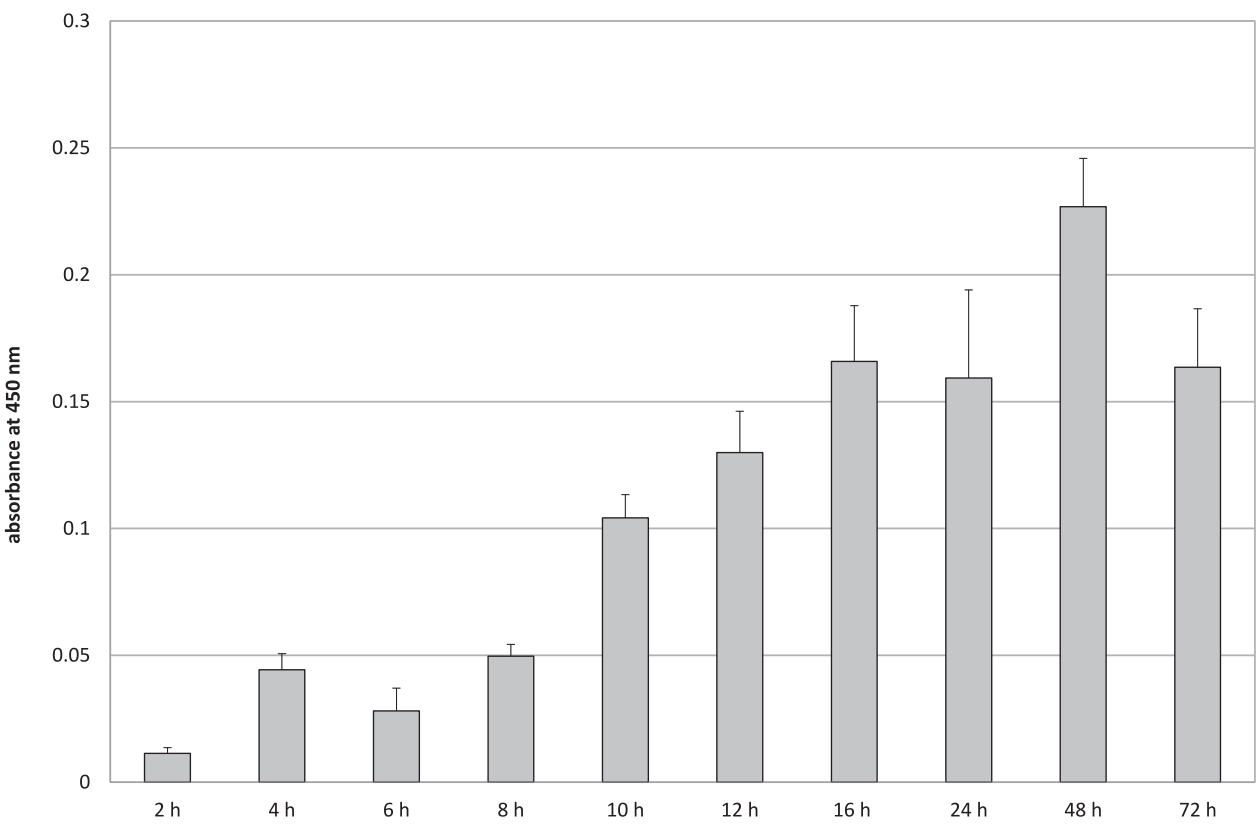

$(* p<0.05)$, very significant $(* * p<0.01)$, and high significant $(* * * p<0.001)$ deviations are indicated.

\section{Results}

\subsection{Time frame of bacterial adherence}

During incubation of $P$. fluorescens ( $P$. fluorescens) on a glass substrate in 1:100 diluted Luria-Bertani medium (LBM), adherence of the microorganisms was already visible after $15 \mathrm{~min}$ (Fig. 1a). The adhering density amplified steadily in the first $120 \mathrm{~min}$ (Fig. 1, b-d) resulting in evenly distributed adhering living bacteria on the surface after $2 \mathrm{~h}$ incubation (Fig. 1e).

Further incubation of $P$. fluorescens for $72 \mathrm{~h}$ resulted in an increase of biofilm biomass during the first $16 \mathrm{~h}$ (Fig. 2). After that incubation time, an equilibrium between growing biomass and detachment of the biomass during medium change was achieved. Additionally, with growing biofilm the metabolism of the basal cells decreases, which may explain decreasing absorbance values after $72 \mathrm{~h}$ incubation. The maximum amount of biomass in this study was detected after $48 \mathrm{~h}$.

\subsection{Nutrient supply}

The influence of nutrient supply on biofilm formation during $72 \mathrm{~h}$ was studied by diluting LBM up to $1: 1000$ in $0.9 \% \mathrm{NaCl}$ (Fig. 3). After $4 \mathrm{~h}$ incubation, the difference in nutrient supply could already be seen with the highest density of adhering microorganisms in undiluted medium and a decreasing density of microorganisms with rising dilution of the medium. In undiluted LBM with high nutrient supply, a strong bacterial growth was observed with biofilm floats and excessive formation of mucus. Incubation with medium nutrient supply in 1:10 diluted LBM lead to slow growth and formation of a stable biofilm on the material surface without excessive mucus formation. Further nutrient reduction in 1:100 and 1:1000 diluted LBM resulted in very slow bacterial growth with no macroscopically visible biofilm formation after $72 \mathrm{~h}$. 
Fig. 3 Influence of nutrient supply on biofilm formation of $P$. fluorescens during $72 \mathrm{~h}$ incubation. LBM was applied undiluted, 1:10, 1:100, and 1:1000 diluted in $0.9 \% \mathrm{NaCl}$. Staining of the biofilm was done with PFA/PI

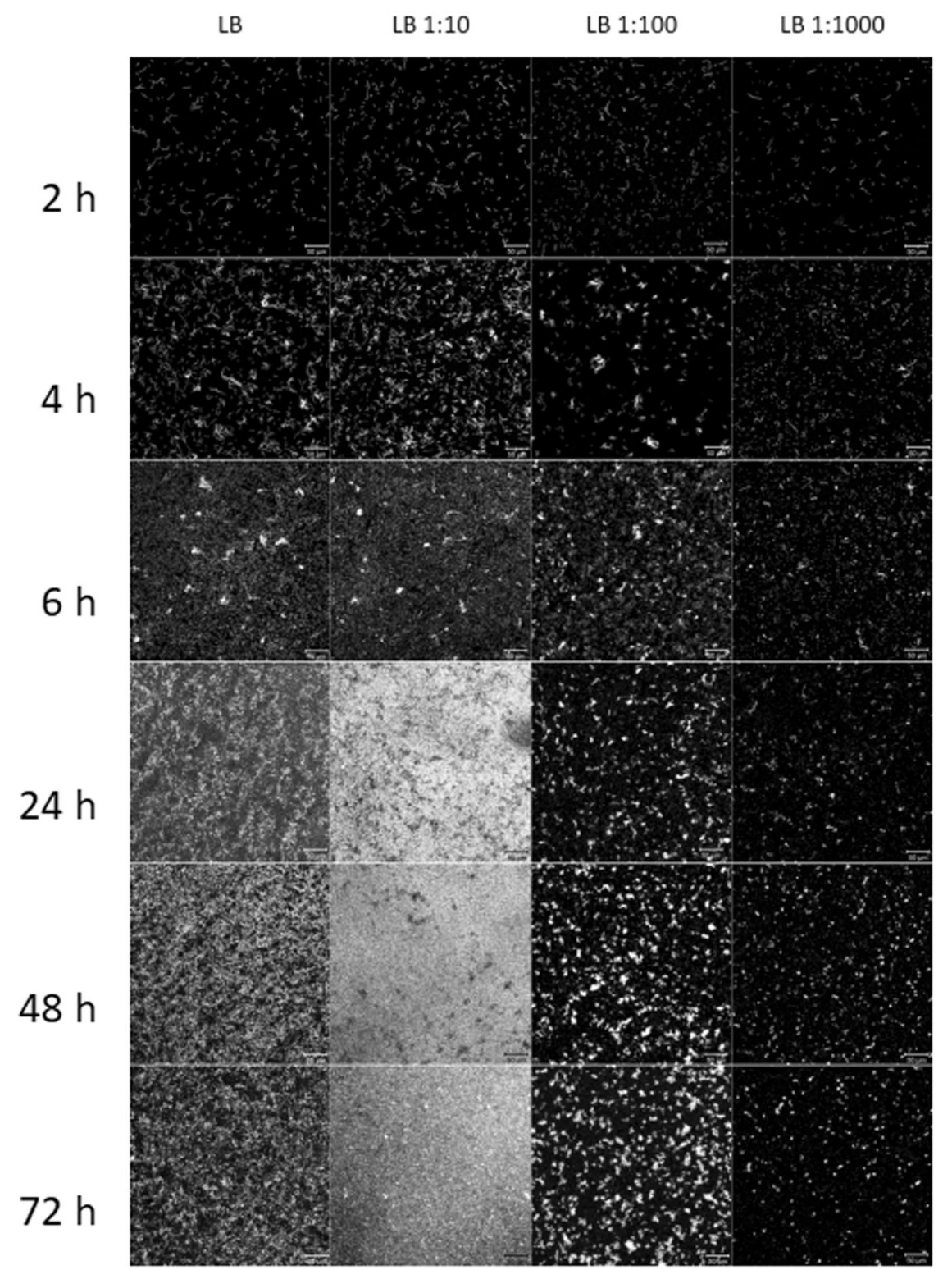

\subsection{Inoculum concentration}

The inoculum concentration plays an important role in the adherence of bacteria in the first $4 \mathrm{~h}$ of incubation. In this time, the inoculum concentration corresponded with the bacteria quantified on the substrate with a significantly different number of living bacteria isolated from biofilms with inoculum concentrations of $10^{3}, 10^{4}, 10^{5}$, and $10^{6} \mathrm{CFU} / \mathrm{mL}$ (Fig. 4). After $24 \mathrm{~h}$, influence of the initial microbial concentration equalized and no considerable differences in bacteria count were detectable at 24,48 , and $72 \mathrm{~h}$ using different inoculum concentrations.

\subsection{Fluid management}

Using a wobble shaker to simulate dynamic growth conditions and to increase the shear stress, a reduced amount of adhering bacteria of $P$. fluorescens was observed compared to static conditions (Fig. 5). This effect was most evident after $48 \mathrm{~h}$ incubation.

In another experiment for mimicking shear stress, the samples were rinsed with LBM before quantification and before medium change after 24 and $48 \mathrm{~h}$. As demonstrated in Fig. 6, a significant reduction of biomass was detected when introducing a rinsing step during static incubation. 
Fig. 4 Influence of inoculum concentration on biofilm formation of $P$. fluorescens during $72 \mathrm{~h}$ incubation in LBM on borofloat coverslips. Inoculum concentrations of $10^{3}$, $10^{4}, 10^{5}$, and $10^{6} \mathrm{CFU} / \mathrm{mL}$ were compared. Biofilm was quantified by colony forming units after biofilm separation and serial dilution. Data are given as mean \pm standard deviation
Fig. 5 Influence of static and dynamic conditions on formation of $P$. fluorescens biofilm during $72 \mathrm{~h}$ incubation in LBM in 96-well MTP. Dynamic conditions were created with a wobble shaker at $200 \mathrm{rpm}$. Medium was changed after 24 and $48 \mathrm{~h}$. Biofilm was quantified by colony forming units after biofilm separation and serial dilution as total CFU/well. Data are given as mean \pm standard deviation
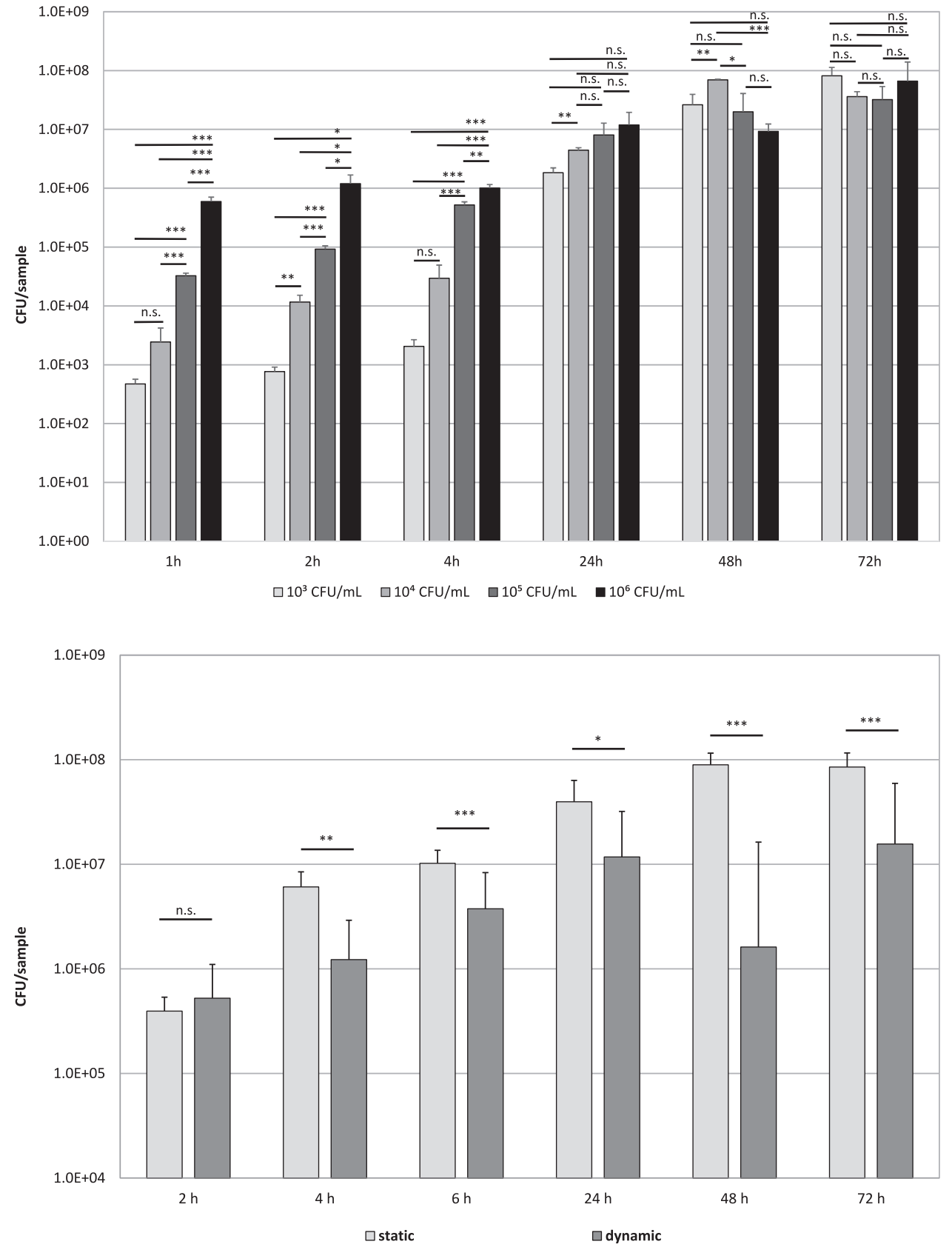

\subsection{Material properties}

Incubation of $P$. fluorescens on borofloat glass, Nunc ${ }^{\mathrm{TM}}$ Thermanox ${ }^{\mathrm{TM}}$ polyester, and poly-L-lysin coated coverslips in 1:100 LBM pointed out the influence of the material on biomass formation (Fig. 7). The highest biomass at each time point was detected using borofloat glass with a maximum after $48 \mathrm{~h}$ incubation. Poly-L-lysin coated coverslips showed a reduced formation of biofilm compared to the other materials at 24 and $72 \mathrm{~h}$ of incubation, the maximum biomass was quantified at $48 \mathrm{~h}$. Incubation of $\mathrm{Nunc}^{\mathrm{TM}}$
Thermanox ${ }^{\mathrm{TM}}$ polyester with $P$. fluorescens resulted in a decreasing biomass during the incubation period.

\subsection{Sample treatment for visualization}

Sample handling turned out as a critical step for visualization of in-vitro biofilm. Initial formation of a biofilm is vulnerable to drying processes, which could lead to misinterpretation of the adhering process. Removal of liquid during staining and washing of the whole MTP enabled individual wells to fall dry for a short time. Hence, 
Fig. 6 Influence of a rinsing step biofilm during $72 \mathrm{~h}$ incubation in LBM, quantified by WST- 1 . The biofilm was rinsed once with LBM before quantification and during medium change after 24 and $48 \mathrm{~h}$. Data are given as mean \pm standard deviation on formation of $P$. fluorescens

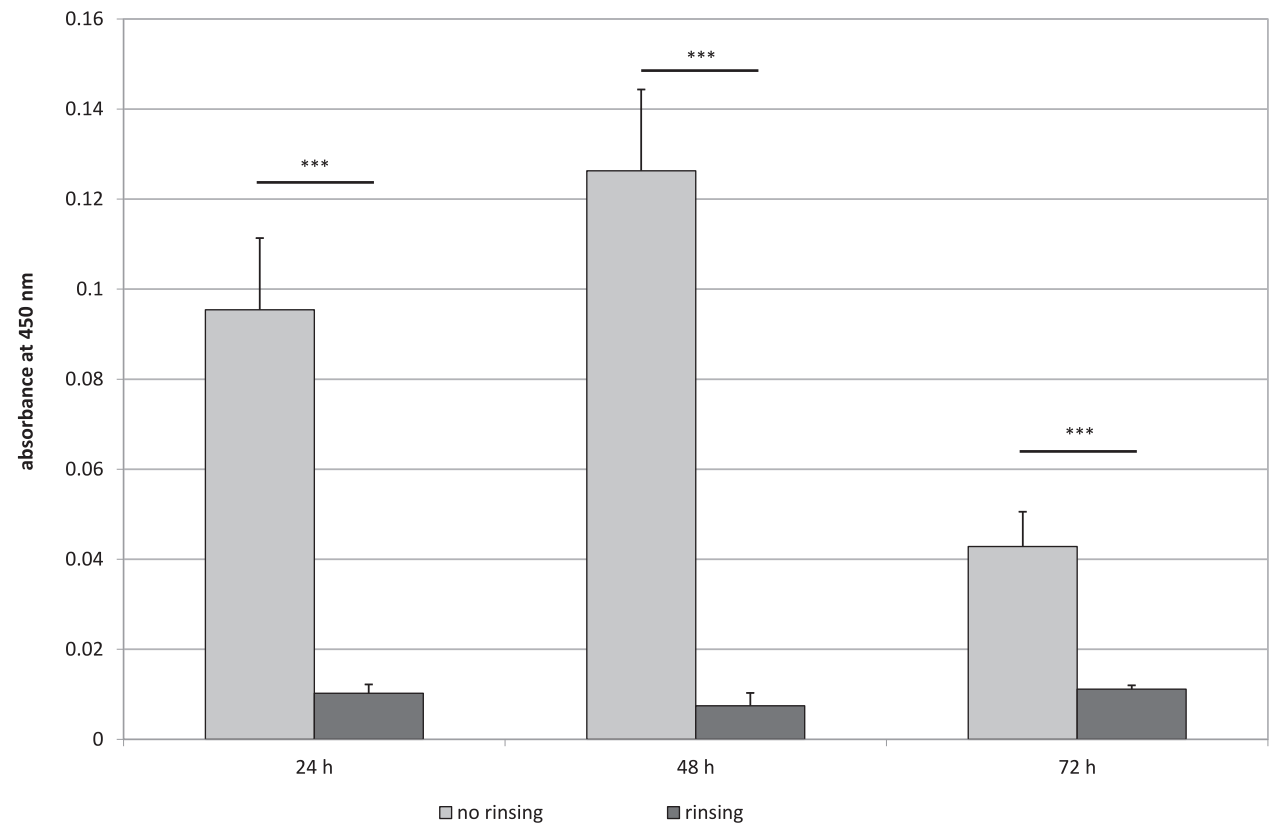

Fig. 7 Influence of the substrate on formation of $P$. fluorescens biofilm during $72 \mathrm{~h}$ incubation on borofloat glass (BF), Nunc ${ }^{\mathrm{TM}}$ Thermanox $^{\mathrm{TM}}$ polyester (TPE), and poly-L-lysin coated coverslips (PLL) in 1:100 LBM. Biofilm mass was quantified by crystal violet staining and subsequent absorbance measurement at $590 \mathrm{~nm}$. Data are given as mean \pm standard deviation

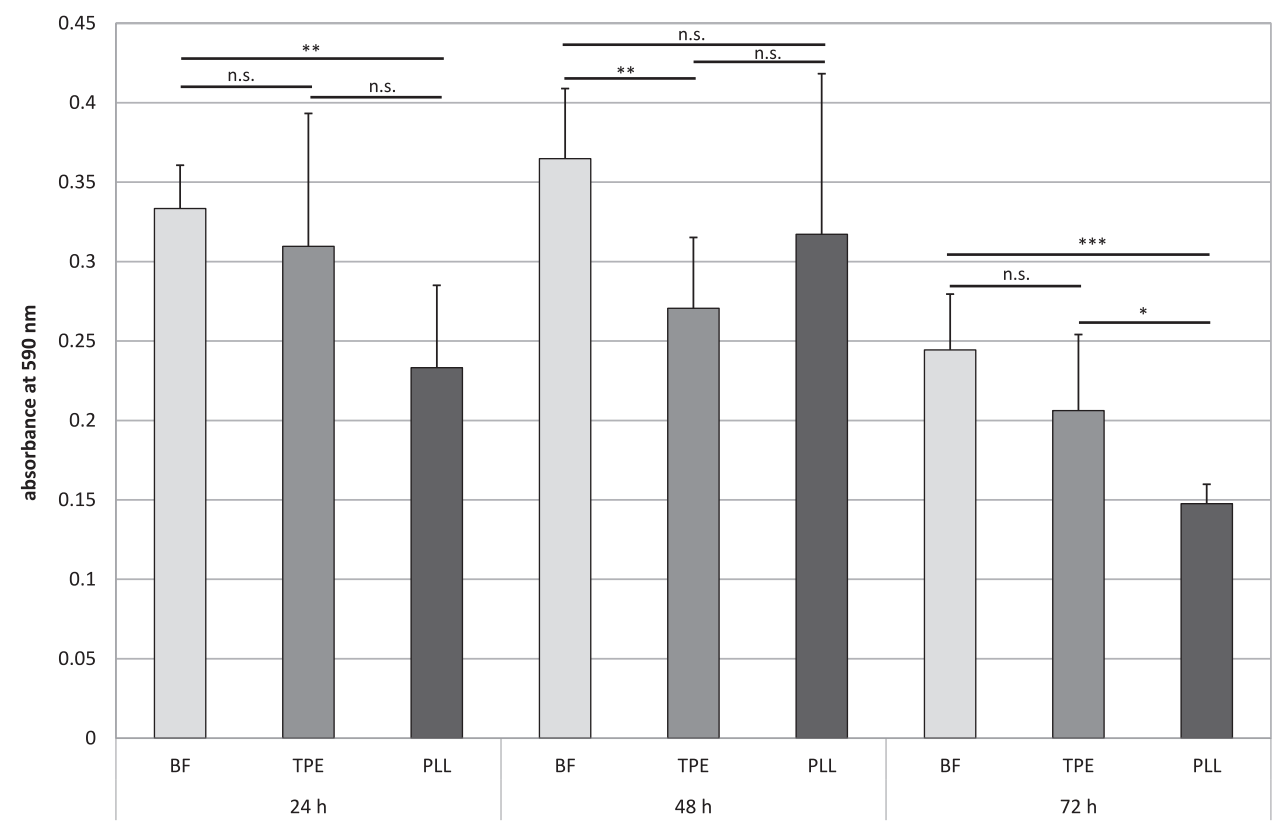

microbial islands were observed when neglecting moisture management during sample preparation (Fig. 8a). This effect could be avoided by treating each well separately. In this way, samples were kept consequently with a liquid film, which resulted in an even distribution of microbes on the surface (Fig. 8b).

\section{Discussion}

Cultivation of biofilms on material surfaces is influenced by a variety of experimental conditions. The design of biofilm experiments for material research has to be optimized with regard to the particular research task and critical parameters in this process have to be considered. In this study, the influence of different factors on biofilm formation was examined i.e. experimental time frame, nutrient supply, inoculum concentration, static and dynamic conditions, material properties, and sample treatment.

In aqueous medium, formation of a biofilm starts almost immediately by conditioning of the substrate with a layer of organic and inorganic particles [12]. This layer alters surface charge, potential and tension of the substrate and provides favorable conditions for bacterial anchorage [13]. 
Fig. 8 Influence of sample treatment on visualization of a 24 h P. fluorescens biofilm incubated on borosilicate glass coverslips in LBM 1:100 diluted in $0.9 \% \mathrm{NaCl}$. Whereas drying of the substrate during incubation and staining procedures with SYTO9/PI/ CFW was partially allowed (a), it was consequently avoided in (b) during staining

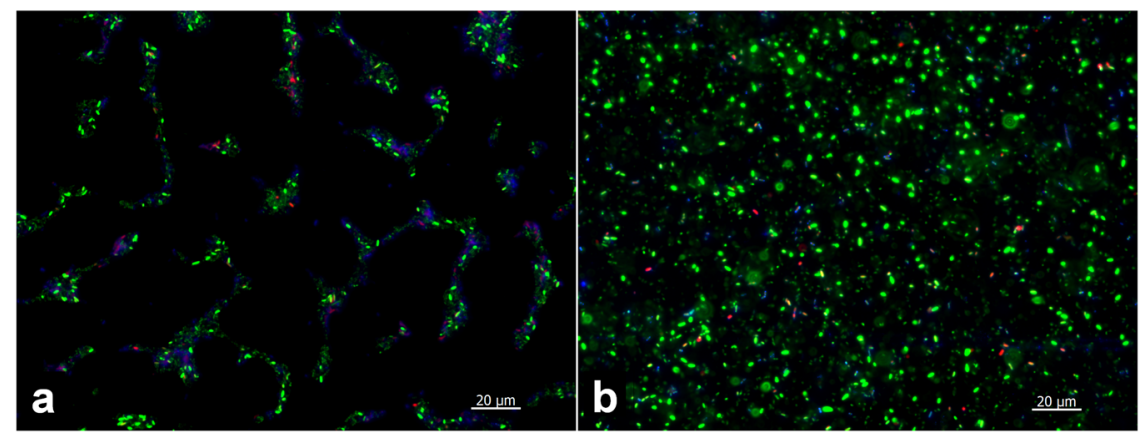

After reversible adhesion of planktonic microbial cells to the conditioned surface, a number of cells remain immobilized and become irreversibly adsorbed. These sessile cells start dividing and a rapid exponential growth phase occurs, often resulting in mushroom-like structures. The production of EPM increases bonding between the cells and imparts mechanical stability of the biofilm. The composition of the matrix is species- and surface-dependent. It is a complex of secreted polysaccharides, proteins, nucleic acids from lysed cells, and absorbed nutrients and ions from the surrounding [2]. In this environment, microbes are effectively protected from harmful external conditions like antibiotics or desiccation.

In our study, adhesion of $P$. fluorescens on borosilicate glass substrate is already observed after $15 \mathrm{~min}$ incubation. Due to rinsing processes during sample preparation and staining, only firmly bound microbes were observed microscopically. The initial transport of microorganisms towards the substratum surface followed by adhesion is very fast. Different mechanisms play an important role in this process, like Brownian motion, gravitation, and diffusion [14]. Crouzet et al. observed a similar timeline in initial biofilm formation by $P$. aeruginosa with attached microbes after $20 \mathrm{~min}$ incubation [15]. After $2 \mathrm{~h}$ incubation, an evenly distributed layer of bound microorganisms was detected in our study. This experimental period of initial adhesion has to be considered for research focusing on e.g. material development studies to prevent initial adhesion of microorganisms to surfaces. Metabolic activity of the $P$. fluorescens biofilm in our experiments increased over a period of $16 \mathrm{~h}$, reaching a plateau of accumulation after 16-24 h. These values are comparable with model systems described in the literature, where the plateau of accumulation in biofilms is achieved after 24 [16], 48 [17], or $72 \mathrm{~h}$ [18]. In the stationary phase of a mature biofilm, the rate of cell division equals the rate of cell death [13]. Enzymes like alginate lyase in the case of $P$. fluorescens are produced by the accumulated biofilm for polysaccharide breakdown and release of surface bacteria, which can colonize additional surfaces [13]. The timeline to reach this plateau of accumulation and the final composition of the extracellular matrix is dependent on species, nutrient availability and fluidic conditions [19]. When working with mature biofilms to investigate e.g. biofilm eradication strategies, it is important to determine the timeline when the plateau of accumulation is reached under the respective conditions to create a reproducible, controllable experimental biofilm.

The influence of nutrient supply on biofilm formation with regard to timeline and quality is crucial in material research. In our study, incubation in undiluted medium with high nutrient load resulted in a strong bacterial growth with biofilm floats and excessive formation of mucus. High nutrient availability in the medium leads to high cell growth rates followed by excessive mucus production and breakdown of the biofilm by enzymatic and mechanical processes [13]. Our results are in accordance with biofilm formation studies with Acinetobacter baumannii, where higher nutrient concentrations decreased biofilm accumulation and poor nutrient media increased biofilm production [20]. Studies on the influence of nutrient supply on biofilm formation in a microchannel system by Liu et al. demonstrated a strong nutritional effect on biofilm morphology [21]. With high nutrient load, the formation of thick, but loose structures was observed, that are highly sensitive to shear stress. In our study, best outcomes with formation of a stable biofilm on the material surface and without excessive mucus production were observed by using 1:10 diluted LB medium. Further reduction of nutrients resulted in slow bacterial growth and little formation of EPM. Kroukamp et al. [22] also demonstrated that a higher nutrient concentration leads to more biomass accumulation. However, this larger biomass is prone to more sloughing, which increases the variability of the biofilm biomass after the first sloughing events. The nature and amount of nutrients present may influence the morphology of bacteria, their surface, and adherence. In oligotrophic, aquatic environments with nutrient stress, organisms respond with enhanced adherence [19]. Therefore, a species dependent selection of medium and medium concentration is important for producing experimental biofilms.

Inoculum cell density plays an important role with regard to biofilm adherence studies. In our experiments, the 
influence of increasing inoculum concentrations on quantification of $P$. fluorescens in the biofilm was detectable only in the adherence phase of $4 \mathrm{~h}$. After $24 \mathrm{~h}$ incubation, no considerable difference was observed in biomass formation with different initial numbers of microbes. In literature, inoculum concentrations in biofilm models vary to a large degree. For Staphylococcus epidermidis biofilm models in 96-well plates, a range from $10^{3}$ to $10^{9} \mathrm{CFU} / \mathrm{mL}$ was reported [23]. Cotter et al. observed a concentrationdependent consumption of dissolved oxygen in the medium in a static 96-well-plate model, resulting in a limited growth at high cell concentrations and alignment of biofilm formation after $4-6 \mathrm{~h}$, independent from starting inoculum concentration [23]. Cell density-sensing molecules were described for A. baumannii and other bacterial pathogens $[24,25]$ for communication between bacterial cells during biofilm formation. These signaling molecules are part of the intercellular communication, known as quorum sensing, that regulates a number of processes during biofilm formation and maturation, like initial formation, control of population size, virulence, defending against other competitive microorganisms, avoiding toxic materials, and detachment $[2,12,26]$. When a plateau of accumulation in the biofilm is reached, in our studies after $16-24 \mathrm{~h}$ incubation, the number of microbes is balanced, independently from starting inoculum concentration.

Fluid management and rinsing procedures have strong influence on adherence, amount and shape of biofilm formation, and detachment processes [3, 27]. This could be confirmed in our studies, where rinsing and shaking reduced biomass formation. Fluid management in experimental biofilms should be adjusted to the research task, the material in question and to the natural occurrence of the biofilm investigated. A variety of biofilm models exist that differ in fluid management. Biofilm formation in MTP is the most popular model [10]. In this model, biofilm is grown in static conditions in the plate or on substrates placed into the plate. The main advantages of this user-friendly, straightforward model are low costs because of small volumes and little technical efforts. This system is ideal for screening purposes regarding antibiofilm substances, substrate modifications, or varying multiple parameters like growth media or atmosphere [9]. Because this model is batch reactor-like, the environment in the wells will change during the experiment, as nutrients are depleted and signaling molecules accumulate, unless the fluid is regularly replaced. Shear stress can only be applied to the system by shaking and rinsing. Many real-life biofilms are exposed to flow conditions and have constant supply of fresh nutrients [28]. So, other investigations may require specific hydrodynamic conditions, substratum composition, or large quantities of biomass. A number of flow displacement models exist with varying shear stress and nutrient distribution, like the modified
Robins device, the Centers for Disease Control biofilm reactor, commercially available flow cells, drip flow reactors, or the rotating disc reactor [9]. In these "open" systems nutrients are continuously added and waste-products are removed.

To increase shear stress in MTP models, plates can be agitated. In our studies, increasing shear stress by shaking resulted in decreasing biomass compared to static cultures. The same effect was shown by Jiang et al. where increasing agitation at high speed enhanced dispersal of biofilms [29]. Similar results were also obtained for Salmonella spp. biofilms incubated in 96-well plates, where biofilm formation was significantly reduced by shaking [30]. In contrast, Donné et al. reported an increase in biofilm biomass with slow horizontal shaking at $25 \mathrm{rpm}$ compared to incubation without shaking of Escherichia coli after $72 \mathrm{~h}$ [2]. They postulate a better biofilm growth at slow shaking speed due to a better nutrient and oxygen dispersion. Therefore, shaking velocity has to be carefully adjusted when agitating MTP models to obtain optimal biofilm growth.

Another influential factor on initial attachment and growth of biofilms are of course the material surface properties. Especially with regard to the major health complications following indwelling medical device infections, investigation into smart materials and coatings that can reduce bacterial attachment is the focus of modern antibiofilm strategies [31]. A number of material properties, like chemical composition, surface charge, roughness, hydrophobicity, or stiffness, influence the biofilm behavior [2, 31]. It was observed, that bacterial attachment accelerates with increasing roughness [12]. By tuning the hydrophobicity of a surface, bacterial adhesion can be either promoted or inhibited, because the preference of surface hydrophobicity differs among the bacterial species [31]. In our studies, differences of biofilm formation were seen between borofloat glass, Nunc ${ }^{\mathrm{TM}}$ Thermanox ${ }^{\mathrm{TM}}$ polyester, and poly-L-lysin coated coverslips with the highest biomass grown on borofloat glass. All tested materials are hydrophilic. Borofloat glass and Nunc ${ }^{\mathrm{TM}}$ Thermanox ${ }^{\mathrm{TM}}$ polyester are negatively charged in contrast to positively charged poly-L-lysin coated coverslips. The surface charge could be the reason for the observed differences in microbial adhesion.

Visualization of microbes adhering to surfaces is a very informative tool for studying biofilm formation processes. To reflect reality during this process we found that sample handling is very important. Especially in the initial state of biofilm formation, the organisms are very vulnerable to drying processes and observed "islands" could lead to misinterpretation of the adhering behavior. To overcome this problem, samples must be kept sufficiently moist during the entire sample preparation and dyeing processes. To our knowledge, this effect caused by sample handling during staining processes of initially adhering biofilms is not yet described in literature. 


\section{Conclusion}

Experimental biofilms are very important tools for understanding the underlying mechanisms of biofilm formation on material surfaces under controlled conditions. For designing intelligent medical biomaterials, these model systems can be successfully used to promote the effective fight against biofilms. In this study, a number of critical factors for cultivation of experimental biofilms in material research are pointed out to help in designing optimal experimental biofilm setups.

\section{Data availability}

All outcome data are available as representative images in the main text. The raw datasets generated and analysed during the current study are available from the corresponding author on reasonable request.

Author contributions $\mathrm{KR}, \mathrm{AG}, \mathrm{STM}, \mathrm{AE}, \mathrm{TJD}, \mathrm{KM}$, and $\mathrm{CW}$ conceived and designed the experiments. KR, AG, STM, AE, TJD, and KM. performed the experiments and collected the data. KR initially drafted the manuscript. KR, AG, BWS, AE, TJD, KM, and CW critically reviewed the paper and approved the final manuscript.

Funding Open Access funding enabled and organized by Projekt DEAL.

\section{Compliance with ethical standards}

Conflict of interest The authors declare no competing interests.

Publisher's note Springer Nature remains neutral with regard to jurisdictional claims in published maps and institutional affiliations.

Open Access This article is licensed under a Creative Commons Attribution 4.0 International License, which permits use, sharing, adaptation, distribution and reproduction in any medium or format, as long as you give appropriate credit to the original author(s) and the source, provide a link to the Creative Commons license, and indicate if changes were made. The images or other third party material in this article are included in the article's Creative Commons license, unless indicated otherwise in a credit line to the material. If material is not included in the article's Creative Commons license and your intended use is not permitted by statutory regulation or exceeds the permitted use, you will need to obtain permission directly from the copyright holder. To view a copy of this license, visit http://creativecommons.org/licenses/by/4.0/.

\section{References}

1. Costerton JW, Cheng KJ, Geesey GG, Ladd TI, Nickel JC, Dasgupta M. et al. Bacterial biofilms in nature and disease. Annu Rev Microbiol. 1987;41:435-64. https://doi.org/10.1146/annurev.mi. 41.100187.002251.

2. Donné J, Dewilde S. The challenging world of biofilm physiology. Adv Micro Physiol. 2015;67:235-92. https://doi.org/10. 1016/bs.ampbs.2015.09.003.

3. Kokare CR, Chakraborty S, Khopade AN, Mahadik KR. Biofilm: importance and applications. Indian J Biotechnol. 2009;8:10.
4. Dufour D, Leung V, Lévesque CM. Bacterial biofilm: structure, function, and antimicrobial resistance. Endod Top. 2010;22:2-16. https://doi.org/10.1111/j.1601-1546.2012.00277.x.

5. NIH, Health NIo, Services. DoHaH. Immunology of biofilms. 2007. http://grants.nih.gov/grants/guide/pa-files/PA-07-288.html.

6. Malone M, Bjarnsholt T, McBain AJ, James GA, Stoodley P, Leaper D. et al. The prevalence of biofilms in chronic wounds: a systematic review and meta-analysis of published data. J Wound Care. 2017;26:20-5. https://doi.org/10.12968/jowc.2017.26.1.20.

7. Dellimore KH, Helyer AR, Franklin SE. A scoping review of important urinary catheter induced complications. J Mater SciMater M. 2013;24:1825-35. https://doi.org/10.1007/s10856-0134953-y.

8. Gottenbos B, Busscher HJ, van der Mei HC, Nieuwenhuis P. Pathogenesis and prevention of biomaterial centered infections. J Mater Sci-Mater M. 2002;13:717-22. https://doi.org/10.1023/A: 1016175502756

9. Coenye T, Nelis HJ. In vitro and in vivo model systems to study microbial biofilm formation. J Microbiol Meth. 2010;83:89-105. https://doi.org/10.1016/j.mimet.2010.08.018.

10. Azeredo J, Azevedo NF, Briandet R, Cerca N, Coenye T, Costa AR. et al. Critical review on biofilm methods. Crit Rev Microbiol. 2017;43:313-51. https://doi.org/10.1080/1040841x.2016.1208146.

11. Coenye T, Kjellerup B, Stoodley P, Bjarnsholt T. The future of biofilm research-Report on the '2019 Biofilm Bash'. Biofilm. 2020;2:100012. https://doi.org/10.1016/j.bioflm.2019.100012.

12. Donlan RM. Biofilms: microbial life on surfaces. Emerg Infect Dis. 2002;8:881-90. https://doi.org/10.3201/eid0809.020063.

13. Garrett TR, Bhakoo M, Zhang Z. Bacterial adhesion and biofilms on surfaces. Prog Nat Sci. 2008;18:1049-56. https://doi.org/10. 1016/j.pnsc.2008.04.001.

14. Bos R, van der Mei HC, Busscher HJ. Physico-chemistry of initial microbial adhesive interactions-its mechanisms and methods for study. FEMS Microbiol Rev. 1999;23:179-230. https://doi.org/ 10.1111/j.1574-6976.1999.tb00396.x.

15. Crouzet M, Claverol S, Lomenech AM, Le Sénéchal C, Costaglioli P, Barthe C. et al. Pseudomonas aeruginosa cells attached to a surface display a typical proteome early as $20 \mathrm{~min}$ of incubation. PLoS One. 2017;12:e0180341. https://doi.org/10.1371/journal.pone.0180341.

16. Shih P-C, Huang C-T. Effects of quorum-sensing deficiency on Pseudomonas aeruginosa biofilm formation and antibiotic resistance. J Antimicrob Chemoth. 2002;49:309-14. https://doi.org/10. 1093/jac/49.2.309.

17. Córdova-Alcántara IM, Venegas-Cortés DL, Martínez-Rivera M, Pérez NO, Rodriguez-Tovar AV. Biofilm characterization of Fusarium solani keratitis isolate: increased resistance to antifungals and UV light. J Microbiol. 2019;57:485-97. https://doi. org/10.1007/s12275-019-8637-2.

18. Runci F, Bonchi C, Frangipani E, Visaggio D, Visca P. Acinetobacter baumannii Biofilm Formation in Human Serum and Disruption by Gallium. Antimicrob Agents Chemother. 2017;61. https://doi.org/10.1128/aac.01563-16.

19. Bowden GH, Li YH. Nutritional influences on biofilm development. Adv Dent Res. 1997;11:81-99. https://doi.org/10.1177/ 08959374970110012101.

20. Eze EC, Chenia HY, El Zowalaty ME. Acinetobacter baumannii biofilms: effects of physicochemical factors, virulence, antibiotic resistance determinants, gene regulation, and future antimicrobial treatments. Infect Drug Resist. 2018;11:2277-99. https://doi.org/ 10.2147/idr.S169894.

21. Liu N, Skauge T, Landa-Marbán D, Hovland B, Thorbjørnsen B, Radu FA. et al. Microfluidic study of effects of flow velocity and nutrient concentration on biofilm accumulation and adhesive strength in the flowing and no-flowing microchannels. J Ind Microbiol Biotechnol. 2019;46:855-68. https://doi.org/10.1007/ s10295-019-02161-x. 
22. Kroukamp O, Dumitrache RG, Wolfaardt GM. Pronounced effect of the nature of the inoculum on biofilm development in flow systems. Appl Environ Microbiol. 2010;76:6025-31. https://doi. org/10.1128/aem.00070-10.

23. Cotter JJ, O'Gara JP, Casey E. Rapid depletion of dissolved oxygen in 96-well microtiter plate Staphylococcus epidermidis biofilm assays promotes biofilm development and is influenced by inoculum cell concentration. Biotechnol Bioeng. 2009; 103:1042-7. https://doi.org/10.1002/bit.22335.

24. Irie Y, Parsek MR. Quorum Sensing and Microbial Biofilms. In: Romeo T, editor. Bacterial Biofilms. Berlin, Heidelberg. Berlin Heidelberg: Springer; 2008. p. 67-84.

25. Gaddy JA, Actis LA. Regulation of Acinetobacter baumannii biofilm formation. Future Microbiol. 2009;4:273-8. https://doi. org/10.2217/fmb.09.5.

26. Bedi B, Maurice N, Sadikot R. Pseudomonas aeruginosa biofilms. JSM Microbiol. 2017;5:11.
27. Buckingham-Meyer K, Goeres DM, Hamilton MA. Comparative evaluation of biofilm disinfectant efficacy tests. J Microbiol Meth. 2007;70:236-44. https://doi.org/10.1016/j.mimet.2007.04.010.

28. Steenackers HP, Parijs I, Foster KR, Vanderleyden J. Experimental evolution in biofilm populations. FEMS Microbiol Rev. 2016;40:373-97. https://doi.org/10.1093/femsre/fuw002.

29. Jiang B, Pei H, Hu W. The Effect of Mechanical Agitation on the Stripping of BioFilm from Ceramic Particles. 4th International Conference on Bioinformatics and Biomedical Engineering. 2010:1-4. https://doi.org/10.1109/ICBBE.2010.5517229.

30. Stepanović S, Ćirković I, Mijač V, Švabić-Vlahović M. Influence of the incubation temperature, atmosphere and dynamic conditions on biofilm formation by Salmonella spp. Food Microbiol. 2003;20:339-43. https://doi.org/10.1016/S0740-0020(02)00123-5.

31. Song F, Koo H, Ren D. Effects of material properties on bacterial adhesion and biofilm formation. J Dent Res. 2015;94:1027-34. https://doi.org/10.1177/0022034515587690. 